\title{
Studying the Electronic Structure of Uranium Dioxide
}

\author{
J.A. Aguiar, $* * * *$ M. Asta, ${ }^{*}$ N. Grønbech-Jensen, ${ }^{* *}$ and N.D. Browning***** \\ * Department of Chemical Engineering and Materials Science, One Shields Avenue, University of \\ California Davis, Davis, CA 95616 \\ ** Department of Applied Science, One Shields Avenue, University of California Davis, Davis, CA \\ 95616 \\ *** Lawrence Livermore National Laboratory, 7000 East Avenue, Livermore, CA 94550
}

Since the inception of nuclear power, issues involving waste control and disposal have posed serious practical, financial, security, health and safety problems, due to the long lifetime and high radiotoxicity of spent nuclear fuel. Under the Global Nuclear Energy Partnership (GNEP), the Department of Energy's Advanced Nuclear Fuel Research and Development program seeks to alleviate these environmental and potential security concerns by efficiently consuming the elements in a new generation of fast-spectrum reactors. These redesigned reactors will transmute the radiotoxic constituents while producing emission-free power. To meet the strict criteria required for safe use in fast reactor technology, several classes of minor actinide containing materials are under consideration, including oxides.

Consequently, there have been growing efforts to apply modern first-principles materials modeling tools as a framework for accelerated design and development of advanced new fuel architectures for fast reactor technology. Following the materials modeling efforts, establishing a damage evolution model for uranium dioxide currently suffers from inaccurate models and a lack of experimental validation. The absence of potential models that accurately address energetic contributions from induced defects suspends the theoretical community. Direct validation of the purposed theoretical techniques, in their application to nuclear fuels, would benefit from direct experimental measurements at the nanometer scale - in particular predictions related to the atomic and electronic structure of relevant point defects and their complexes.

In order to address these deficiencies, we have commenced experimental and theoretical research to characterize irradiated nuclear fuel elements with state of the art microscopy. The sample studied was porous depleted uranium dioxide which was pressed, dimpled, and ion milled into a $3 \mathrm{~mm}$ disc. The experimental technique used to study the sample is high angle annular dark field scanning transmission electron microscopy (STEM) paired with electronic energy loss spectroscopy (EELS). To increase the spatial and energy resolution, the monochromated and $\mathrm{C}_{\mathrm{s}}$ corrected FEI Titan electron microscope at Lawrence Livermore National Laboratory is used for the analysis.

Within the density functional theoretical framework, we employ a variety of calculations, but for the purposes of this presentation the latest local spin density approximation (LSDA+U) with unique materials considerations particular to $\mathrm{UO}_{2}$ is considered. The materials considerations include correlated uranium f-states, antiferromagnetic ordering, spin-orbit coupling, and in view of the direct comparison to STEM/EELS the core-hole effect. To directly compare experiment and theory the core EELS oxygen $K$-edge is chosen since the peak is most energetically sensitive to induced Frenkel defects and vacancies. 
Primarily, the electronic structure calculations assist in unraveling the plausible local atomic contributions on the defect-derived measured oxygen $K$-edge. The hyperstoichiometric theoretical density of states shown in Fig 1 demonstrates an antiferromagnetic insulator with a $1.126 \mathrm{eV}$ bandgap. The magnetic moments on the uranium atoms calculated from spin densities is 1.74 and $1.02 \mu_{\mathrm{B}}$ which corresponds to the $4^{+}$and $5^{+}$states, respectively. The magnetic moments are affected by the vicinity of the interstitial oxygen, but for the impervious uranium 4+ atoms they are in exact agreement with the antiferromagnetic bulk experimental value [2]. Initial results indicate valid current electronic modeling techniques for the primitive non-defect derived single atomic oxygen spectra shown in Fig 2. In later studies, we will simultaneously map the local-binding environment via the oxygen $K$-edge. In the near future, as well, the atomic defect oxygen case will be further investigated. This investigation into density functional theory and electron microscopy for strongly correlated materials will be presented.

References

[1] This research was supported by the US Department of Energy Nuclear Energy Research Initiative for Consortia (NERI-C) contract number DR-FG07-071D14893.

Y. Yun, H. Kim and K. Park, Nucl Eng Technol 37 (2005), p. 293.
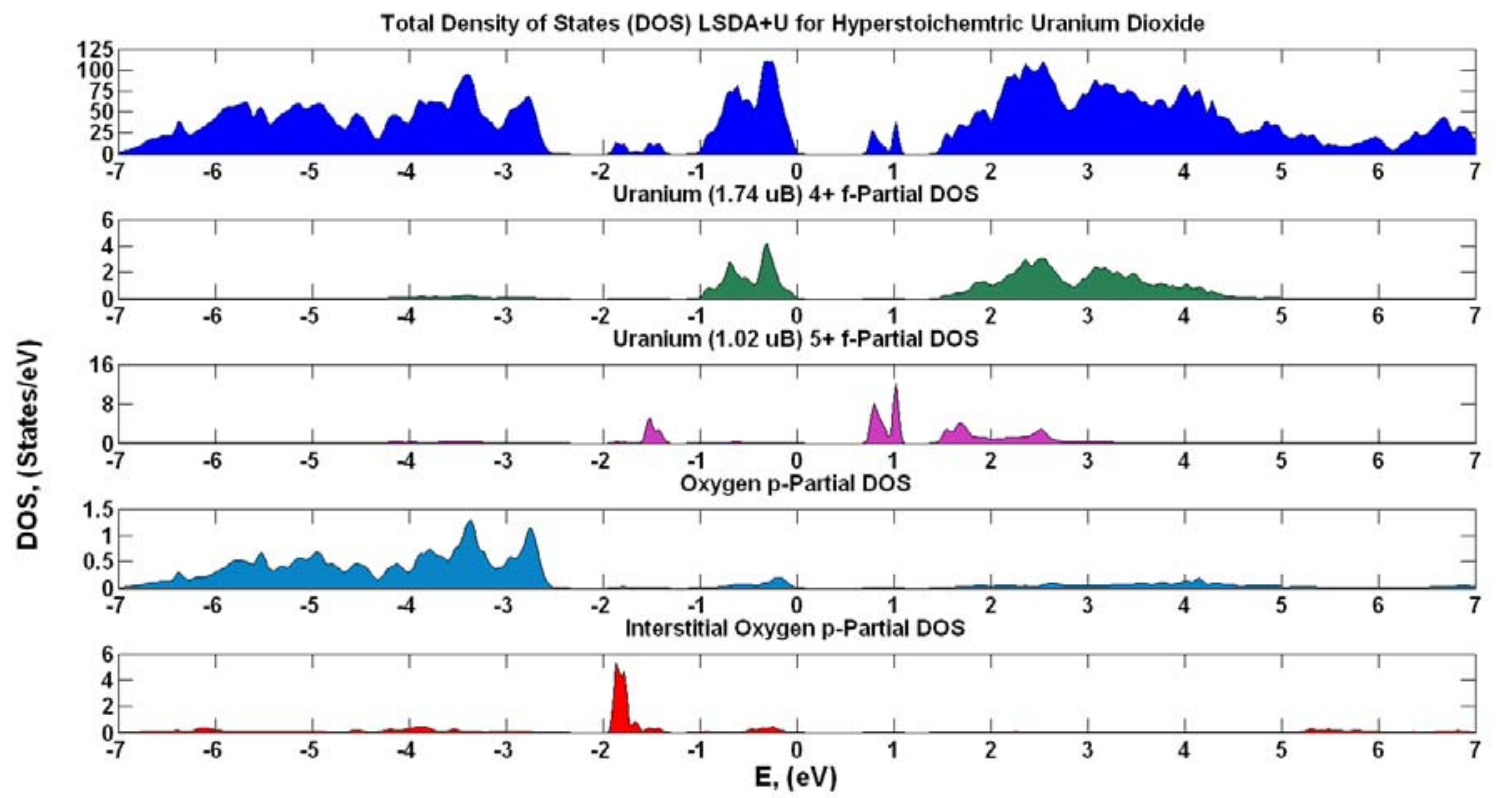

\section{Oxygen K- Edge EELS Profiles for Uranium Dioxide}

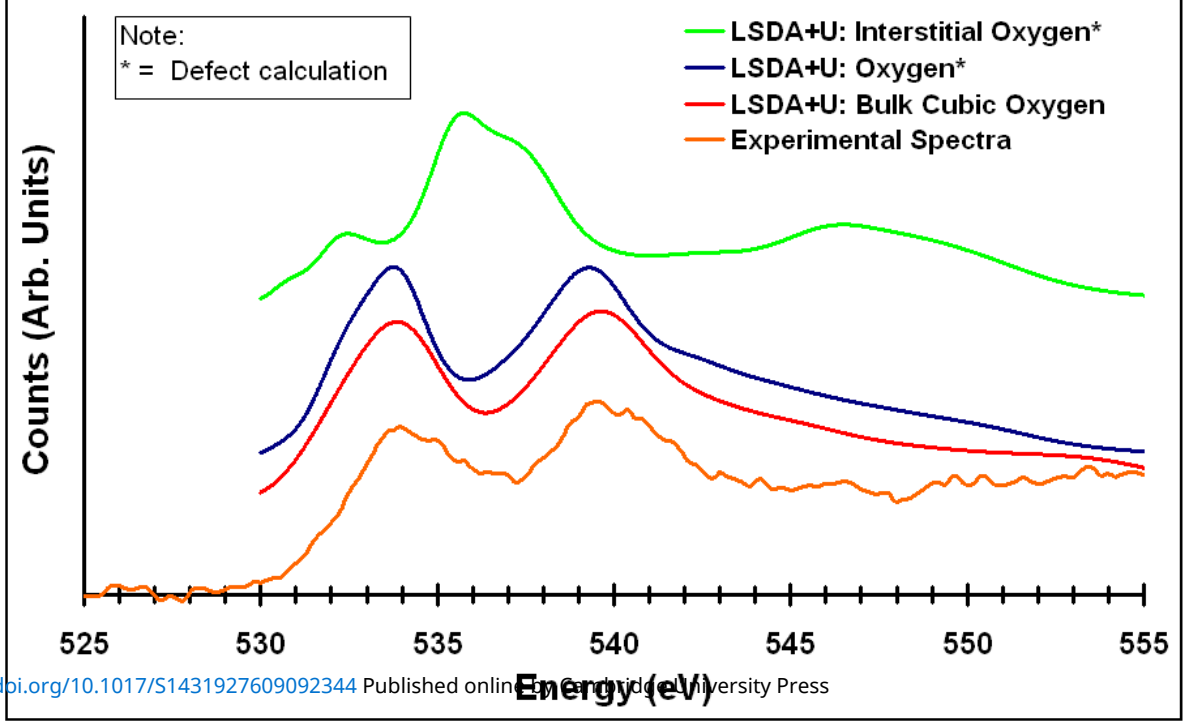

FIG. 1. Local atomic contributions to oxygen $K$-edge outlined in the total and partial density of states in hyperstoichemtric oxide.

FIG.2. Experimental comparison for $\mathrm{UO}_{2}$ with theoretical oxygen $K$-edge profiles for hyper and stoichiometric configurations. 